\title{
Crystal structure of atypical ubiquitin ligase PCAF_N
}

\section{Sachiko Toma-Fukai ${ }^{1}$, Ryota Hibi ${ }^{2}$, Takao Naganuma ${ }^{3}$, Mashito Sakai ${ }^{3}$, Shinya Saijo ${ }^{4}$, Nobutaka Shimizu ${ }^{4}$, Michihiro Matsumoto ${ }^{3}$, Toshiyuki Shimi ${ }^{2}$}

\author{
${ }^{I}$ Nara Institute of Science and Technology; \\ ${ }^{2}$ The University of Tokyo; \\ ${ }^{3}$ National Center for Global Health and Medicine; \\ ${ }^{4}$ High Energy Accelerator Research Organization (KEK) \\ toma@ms.naist.jp
}

Post-translational modification of proteins regulates many biological processes. Acetyltransferase transfers acetyl groups to lysine residues on target proteins and is a major type of post-translational enzyme. General control nonderepressible5 (GCN5, also known as Kat2a) is one of the histone acetyltransferases that promote transcriptional activity.

Metazoans possess two GCN5 isoforms that arise from alternative splicing. The lower molecular weight isoform (isoform2) is similar in size and function to yeast GCN5, consisting of an acetyltransferase (AT) domain and a bromodomain at the N and C termini, respectively. Another higher molecular weight isoform (isoform 1) has an N-terminal extension. The amino acid sequence of this Nterminal extension is similar to the N-terminal domain of p300/CBP-associated factor (PCAF, also known as Kat2b) and is conserved among vertebrates. This $\mathrm{N}$-terminal domain is called the PCAF_N domain.

Ubiquitination is also a post-translational modification that targets lysine residues. This modification regulates many cellular processes, including cell division and immune responses, among others. Ubiquitination is catalyzed by the sequential reaction of ubiquitin-activating enzyme (E1), ubiquitin-conjugating enzyme (E2), and ubiquitin ligase (E3), which is responsible for the ligation of ubiquitin onto a substrate in conjunction with the E2.

A previous study reported that PCAF also harbors ubiquitination activity in addition to acetyltransferase activity. PCAF_N is identified as a domain that contains a ubiquitin E3 ligase activity. Although the longer isoform of GCN5 possesses the PCA $\bar{A} F$ _N domain, it has not been revealed whether GCN5 functions as an E3 enzyme.

We demonstrated that GCN5 exhibited ubiquitination activity and its activity was supported by the ubiquitin-conjugating enzyme UbcH5. Moreover, we determined the crystal structure of the PCAF_N domain at $1.8 \AA$ resolution and found that the PCAF_N domain folds into a helical structure with a characteristic binuclear zinc region, which could not be predicted from sequence analyses. The zinc region is distinct from known E3 ligase structures, suggesting this region may form a new class of E3 ligase. Our biochemical and structural study provides valuable insight into not only the functional significance of GCN5 but also into ubiquitin biology.

Keywords: ubiquitin ligase $x$-ray crystallography 